\title{
Computational Assessment of Blood Flow Heterogeneity in Peritoneal Dialysis Patients' Cardiac Ventricles
}

\author{
Sanjay R. Kharche ${ }^{1,2 \star}$, Aaron So ${ }^{2,3}$, Fabio Salerno ${ }^{1}$, Ting-Yim Lee ${ }^{3}$, Chris Ellis ${ }^{2}$, \\ Daniel Goldman ${ }^{2}$ and Christopher W. Mclntyre ${ }^{1,2 *}$ \\ ${ }^{1}$ Kidney Clinical Research Unit, Lawson's Health Research Institute, Victoria Hospital, London, ON, Canada, ${ }^{2}$ Department of \\ Medical Biophysics, Schulich School of Medicine and Dentistry, University of Western Ontario, London, ON, Canada, \\ ${ }^{3}$ Robarts Research Institute, University of Western Ontario, London, ON, Canada
}

OPEN ACCESS

Edited by:

Eun Bo Shim,

Kangwon National University,

South Korea

Reviewed by:

Soroush Safaei,

University of Auckland, New Zealand

Ki Moo Lim,

Kumoh National Institute of

Technology, South Korea

*Correspondence:

Sanjay R. Kharche

sanjay.kharche@/hsc.on.ca

Christopher W. McIntyre

c.w.mcintyre@/hsc.on.ca

Specialty section:

This article was submitted to

Computational Physiology and

Medicine,

a section of the journal

Frontiers in Physiology

Received: 13 January 2018

Accepted: 20 April 2018

Published: 17 May 2018

Citation:

Kharche SR, So A, Salerno F, Lee T-Y, Ellis $C$, Goldman D and Mclntyre CW (2018) Computational Assessment of Blood Flow Heterogeneity in

Peritoneal Dialysis Patients' Cardiac Ventricles. Front. Physiol. 9:511.

doi: 10.3389/fphys.2018.00511
Dialysis prolongs life but augments cardiovascular mortality. Imaging data suggests that dialysis increases myocardial blood flow (BF) heterogeneity, but its causes remain poorly understood. A biophysical model of human coronary vasculature was used to explain the imaging observations, and highlight causes of coronary BF heterogeneity. Post-dialysis CT images from patients under control, pharmacological stress (adenosine), therapy (cooled dialysate), and adenosine and cooled dialysate conditions were obtained. The data presented disparate phenotypes. To dissect vascular mechanisms, a 3D human vasculature model based on known experimental coronary morphometry and a space filling algorithm was implemented. Steady state simulations were performed to investigate the effects of altered aortic pressure and blood vessel diameters on myocardial BF heterogeneity. Imaging showed that stress and therapy potentially increased mean and total BF, while reducing heterogeneity. BF histograms of one patient showed multi-modality. Using the model, it was found that total coronary BF increased as coronary perfusion pressure was increased. BF heterogeneity was differentially affected by large or small vessel blocking. BF heterogeneity was found to be inversely related to small blood vessel diameters. Simulation of large artery stenosis indicates that BF became heterogeneous (increase relative dispersion) and gave multi-modal histograms. The total transmural BF as well as transmural BF heterogeneity reduced due to large artery stenosis, generating large patches of very low BF regions downstream. Blocking of arteries at various orders showed that blocking larger arteries results in multi-modal BF histograms and large patches of low BF, whereas smaller artery blocking results in augmented relative dispersion and fractal dimension. Transmural heterogeneity was also affected. Finally, the effects of augmented aortic pressure in the presence of blood vessel blocking shows differential effects on BF heterogeneity as well as transmural BF. Improved aortic blood pressure may improve total BF. Stress and therapy may be effective if they dilate small vessels. A potential cause for the observed complex BF distributions (multi-modal BF histograms) may indicate existing large vessel stenosis. The intuitive BF heterogeneity methods used can be readily used in clinical studies. Further development of the model and methods will permit personalized assessment of patient BF status.

Keywords: coronary vessels, cardiac physiology, mathematical modeling, computational physiology, integrative physiology 


\section{INTRODUCTION}

Dialysis is a life prolonging treatment but significantly reduces quality of life due to its deleterious side effects on the heart. This mathematical modeling study explores some of the coronary vasculature based causes of myocardial blood flow (BF) heterogeneity.

\section{Clinical Motivation}

The over $20 \%$ mortality among end stage renal disease (ESRD) patients receiving dialysis treatment is often due to cardiovascular complications (Collins et al., 2015). Dialysis is a repetitive sub-lethal ischemia that is known to produce cardiac contractile dysfunction as observed clinically (Burton et al., 2009; McIntyre, 2010; Jefferies et al., 2011; Breidthardt et al., 2012). ESRD patients also experience significantly reduced coronary blood flow (BF) (Dasselaar et al., 2009). The reduced BF may often occur due to calcification (McIntyre et al., 2013; McIntyre and Odudu, 2014) that reduces arterial diameters. Experimental observations have led to the belief that an increased resistance of blood vessels in the sub-endocardium promotes increased transmural BF heterogeneity (Algranati et al., 2011). Although non-invasive interventions may not affect existing large vessel structural defects such as stenosis, it is thought that adenosine stress and dialysate cooling therapy may improve myocardial BF by vasodilation of the smaller blood vessels. The interventions may also improve myocardial BF by improving aortic pressure. The mechanisms by which BF heterogeneity is affected remain unclear. Knowledge of the cause-effect relationships may permit design of future clinical trials and augment the precision of medications given to this critically ill group of patients.

\section{Previous Coronary Vasculature Theoretical Models, Extant Experimental Data}

The predominantly dichotomous mammalian coronary architecture is complex with millions of arterial segments (Kassab et al., 1993, 1997a). The larger left and right coronary arteries ( $\sim 3 \mathrm{~mm}$ diameters, $80 \mathrm{~mm}$ lengths) forming aortic ostia give rise to arterial trees, which together constitute the arterial vasculature. A very large number of pre-capillary arterioles deliver blood to capillary beds $(\sim 0.06 \mathrm{~mm}$ diameters, $0.15 \mathrm{~mm}$ lengths). A spectrum of biophysical and anatomical properties of vasculature has been studied to permit patho-physiological investigations. The topology of coronary arterial trees has been quantified by Kassab et al. (1993, 1997a,b) using silicon elastometer casts. In the absence of biophysical morphometry data, theoretical vasculature topologies can also be generated (Keelan et al., 2016). The topology data obtained from large animal hearts can be scaled to the human heart using clinical angiograms (Dodge et al., 1992). To permit generating a 3D geometry from the topology, the bifurcation properties of the network have been quantified. In accordance with Murray's law (Murray, 1926a,b), the relationship between artery segment lengths, diameters, and bifurcation angles and planes has been described by Zamir and Phipps (1988) and Zamir et al. (1983). Using the topology and Murray's law, algorithms that distributed the topology as uniformly as possible in $3 \mathrm{D}$ space were developed. The deterministic algorithm, which may overall be called "space filling algorithm," is based on the principles of self-avoidance and boundary avoidance and was developed by Beard and Bassingthwaighte (2000). Several studies further developed the space filling algorithm which optimizes vascular spatial distribution as well as delivery of BF to various parts of the heart (notably in Smith et al., 2000; Mittal et al., 2005; Kaimovitz et al., 2010), and have either generated the complete or partial epicardial vasculature networks. Whereas vascular resistance is regulated by geometry alone, the $\mathrm{BF}$ and pressure at each location in the network also depends on the properties of fluid flowing through the network. Blood is a biphasic fluid and alterations of its viscosity, dependent on haematocrit, have been quantified by Pries et al. (1996). The intricate problem of vasculature involves optimizing relative arterial diameters, bifurcation angles, providing of BF to potentially empty regions, and being as widely distributed in the myocardium as possible. In the absence of experimental data it may be possible to generate virtual vasculatures based on biophysical principles and computational optimization of cost functions (Karch et al., 1999; Kaimovitz et al., 2005; Keelan et al., 2016). The scientific problems arising in vasculature modeling based on available knowledge have been addressed in individual theoretical studies with particular aims. Alarcon et al. provide a design principle in light of complex blood rheology (Alarcón et al., 2005).

The effects of structural defects on hemodynamic distribution have been studied by Yang and Wang (2013). The availability of mathematical-computational tools such as the $3 \mathrm{D}$ coronary vasculature models has encouraged the investigation of specific disease conditions in the heart (Zhang et al., 2014). Similar to the present study, a detailed model by Fung et al. $(2010,2011)$ has been developed to assist in evaluation of imaging hearts with perfusion defects. An extension of previous and presented models will incorporate the multi-scale nature of delivery of oxygen (Goldman et al., 2017; Mason McClatchey et al., 2017) to myocardial tissue. Although several studies have characterized the properties of the coronary vasculature, the use of this vast basic science knowledge for clinical purposes remains acutely limited.

In this study, we endeavored to exploit organ level vasculature modeling to investigate potential causes for our clinical myocardial BF heterogeneity observations. This study developed our biophysically informed topology-geometry of the human coronary vasculature.

\section{METHODS}

\section{Clinical Imaging}

It is thought that peritoneal dialysis increases coronary BF heterogeneity in patients. CT imaging was performed to test whether adenosine, cooled dialysate, and adenosine combined with cooled dialysate can enhance myocardial $\mathrm{BF}$, and reduce $\mathrm{BF}$ heterogeneity.

\section{Patient Recruitment}

Three chronic end stage renal failure patients, aged between 58-63 years, were recruited from the London Health Sciences 
Centre Peritoneal Dialysis Program (London Ontario, Canada). Each patient had been on peritoneal dialysis for a minimum of 3 months prior to recruitment. All patients were informed regarding the study, after which written consents were obtained in accordance with the hospital and university procedures. All participants provided informed and written consent. The study protocol was approved by the research ethics board at Western University (London, Ontario, Canada).

\section{Imaging Study Protocol}

Briefly, each patient was scanned four times in two study visits. During the first visit, a glucose based peritoneal dialysate, according to their prescription, was administered at a physiological temperature of $37^{\circ} \mathrm{C}$, after which they were scanned with and without adenosine stress. During the second visit, patients were administered the peritoneal dialysis dose but with a cooled dialysate $\left(32.5^{\circ} \mathrm{C}\right)$, after which they were scanned with and without adenosine stress.

To assist the dynamic CT scanning, a contrast enhancing agent, lopamidol, was administered. The heart rate was reduced using a $\beta$-blocker, metoprolol, that permitted a longer diastolic phase in the left ventricle. The details of the imaging protocol and image processing that computed the BF maps are given in Section S1.

\section{Patient Blood Pressure}

The diastolic blood pressures values ranged between diastolic 71 and $85 \mathrm{mmHg}$, and systolic values between 130 and $210 \mathrm{mmHg}$. Other clinical laboratory measurements were not considered in this study.

\section{D Heart Segmentation}

The $2 \mathrm{D}$ registered $\mathrm{BF}$ map slices were segmented by a physician semi-automatically using Fiji/ImageJ (Schindelin et al., 2012). Each slice was first segmented to remove non-myocardial tissue signals. Subsequently, the image was thresholded to between 0 and $600 \mathrm{ml} / \mathrm{mg} / \mathrm{min}$ to selectively remove residual signals pertaining to intracardiac (left and right ventricle chambers) blood, while preserving signals providing coronary $\mathrm{BF}$ distribution. The slices were stacked to reconstruct each patients BF maps under each of the four clinical conditions (see section Imaging study protocol). The reconstruction was stored in a structured array of $0.5(\mathrm{X}) \times 0.5(\mathrm{Y}) \times 5(\mathrm{Z}) \mathrm{mm}^{3}$ array. The structured array representation was used to quantify $\mathrm{BF}$ heterogeneity (see below).

\section{Model Construction}

\section{Human Ventricle Anatomy to Contain Vasculature Geometry}

A representation of the human ventricles was constructed to permit generation of vascular geometry within it. An idealized representation bound by truncated ellipsoids was constructed (Göktepe and Kuhl, 2010). The dimensions of the anatomy are detailed in Section S2 and Figure S1. The coronary vasculature geometry was generated within the ventricular anatomy.

\section{Topology of Coronary Vasculature Based on Morphometry Biophysical Data}

The porcine coronary vasculature morphometry (Kassab et al., 1993) was used to construct arterial tree topology. The stochastic morphometry data consists of arterial segment connectivity matrices, segment lengths, and segment radii. In this study, two coronary arterial topologies were constructed as binary trees, one each for the right and left coronary arteries. The segments, defined as parts of arteries between two consecutive bifurcation nodes, in the trees were numbered according to the Strahler number (SN) ordering (Strahler, 1957). Within this numbering system the largest arteries are a series of segments of the same SN, namely the left anterior descending (LAD) and the right coronary artery (RCA), have a $\mathrm{SN}$ of 11 . The left circumflex artery (LCX) which has a SN of 10 emerges at a bifurcation of the LAD, and provides $\mathrm{BF}$ to a sub-tree in the left ventricle. Whereas the larger vessels (SN 9-SN 11) are restricted to the epicardial surface, smaller vessels (SN 6-SN 8) provide BF transmurally (Kaimovitz et al., 2005). As a computationally manageable approximation that permitted simulation of whole heart $\mathrm{BF}$, the arterial trees were generated for SN 6-SN 11, where SN 6 was identified based on its diameter and the number of bifurcations that would be needed to reach the capillary level (SN 0). First, arterial elements, defined as a series of connected segments of the same $\mathrm{SN}$, were generated stochastically using the segment to element ratios in the morphometry data (Section S3). The elements were then assembled, again stochastically, using connectivity matrices (Section S3; Kassab et al., 1993) to stochastically generate multiple instances of the whole heart's coronary arterial binary tree topologies. Each element was assigned a constant radius along its length adapted from the experimental data of Kassab et al. (1993) and scaled to human using clinical data (Dodge et al., 1992) in accordance with previous modeling studies (Beard and Bassingthwaighte, 2000; Smith et al., 2000). Finally, the segments were assigned lengths stochastically (Section S3; Kassab et al., 1993). The total tree lengths were bounded to avoid nonphysiologically short or long trees (Kaimovitz et al., 2005). The total tree length of RCA was limited to between 120 and $192 \mathrm{~mm}$, and those of LAD and LCX were both limited to between 100 and $160 \mathrm{~mm}$.

Using the segment lengths (L) and radius ( $\mathrm{r}$ ) information assigned during topology generation, and using a blood viscosity value of $\mu=3.6 \times 10^{-3} \mathrm{~Pa}$ (Keelan et al., 2016), a value of resistance to flow $(\mathrm{R})$ in each segment was assigned using the relationship:

$$
R=\frac{8 \mu L}{\pi r^{4}}
$$

The downstream resistance at any bifurcation node was then computed recursively. Elements that include segments of SN 8SN 11 constitute epicardial vessels (Kassab et al., 1993; Smith et al., 2000; Kaimovitz et al., 2005).

To permit generation of arterial geometry, pressure boundary conditions were imposed on the inlets and terminal nodes of the arterial tree topologies and $\mathrm{BF}$ in each segment and pressure at each internal node were computed recursively. At 
the terminal nodes ( $\mathrm{SN} \mathrm{6}$ ), the pressure was set at $20 \mathrm{mmHg}$. At the inlets, the pressure was set at $100 \mathrm{mmHg}$ under control conditions, and varied to simulate disease, stress, or therapy. The boundary conditions, conservation of flow at bifurcation nodes, and Poiseuille's law for steady state flow, $\Delta \mathrm{P}$ (pressure) $=\mathrm{R}$ (resistance) $\times \mathrm{Q}(\mathrm{BF})$ in each segment and in whole trees, permitted calculation of pressure at each bifurcation node, and $\mathrm{BF}$ through each segment of the arterial trees.

\section{Generation of Arterial Tree Geometry}

Each instance of the arterial tree topology was used to generate a corresponding coronary arterial geometry. The geometry structure was generated using a modification of the method developed by Fung et al. (2011). The method for assigning coordinates to distal nodes of daughter segments is illustrated in Figure 1 which is inspired by Fung et al. (2011).

\section{Placement of RCA, LAD, and LCX elements}

The roots of the RCA and LAD arterial trees were placed approximately at their aortic ostia locations (Smith et al., 2000; Kaimovitz et al., 2005; Keelan et al., 2016). The largest arteries were placed either along the right or left epicardial surfaces (RCA and LCX respectively), or traversing from the base (AV border) to the apex (LAD). Doing so provided a starting point for the self-avoidance and boundary-avoidance algorithms as described by Beard and Bassingthwaighte (2000).

\section{Initial daughter segment branching angles}

The branching of a parent segment into two daughter segments is known to optimally minimize shear. As shown by Hacking et al. (1996) and Zamir (1976a,b), the initial left and right branching angles, $\theta_{L}$ and $\theta_{R}$ respectively (see Figure 1A), were computed using the parent (denoted by subscript "p"), left (denoted by subscript "L"), and right (denoted by subscript "R") radii (r) and BFs (Q) as:

$$
\begin{aligned}
& \theta_{L}=\cos ^{-1}\left[\frac{Q_{p}{ }^{2} / r_{p}{ }^{4}+Q_{R}{ }^{2} / r_{R}{ }^{4}-Q_{L}{ }^{2} / r_{L}{ }^{4}}{2 Q_{p} Q_{L} / r_{p}{ }^{2} r_{L}{ }^{2}}\right] \\
& \theta_{R}=\cos ^{-1}\left[\frac{Q_{p}{ }^{2} / r_{p}{ }^{4}+Q_{L}{ }^{2} / r_{L}{ }^{4}-Q_{R}{ }^{2} / r_{R}{ }^{4}}{2 Q_{p} Q_{R} / r_{p}{ }^{2} r_{R}{ }^{2}}\right]
\end{aligned}
$$

In conjunction with the combined branching vector (see below), these branching angles were used to assign initial locations of daughter segment distal nodes. The combined branching vector is a linear combination of the vascular supply and the boundary avoidance vectors.

\section{Self-avoidance vector}

To permit the child nodes to occupy a location within the tissue that was so far sub-optimally supplied with BF, a self-avoidance vector, $\vec{v}$, was computed as shown in Figure 1B. It was computed using Equation (3) as:

$$
\vec{v}_{s}=\sum_{i} \frac{\left(L_{s} / d_{i}\right)^{\xi}}{1+\left(L_{s} / d_{i}\right)^{\xi}} \frac{\vec{s}_{i}}{d_{i}}
$$

A Assignment of coordinates Initial daughter segment branching angles

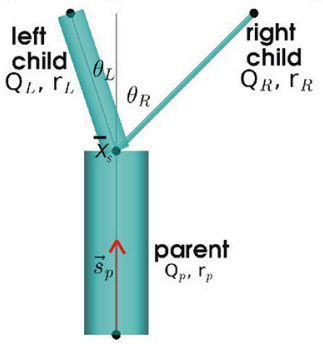

B Self-avoidance vector C Boundary avoidance vector
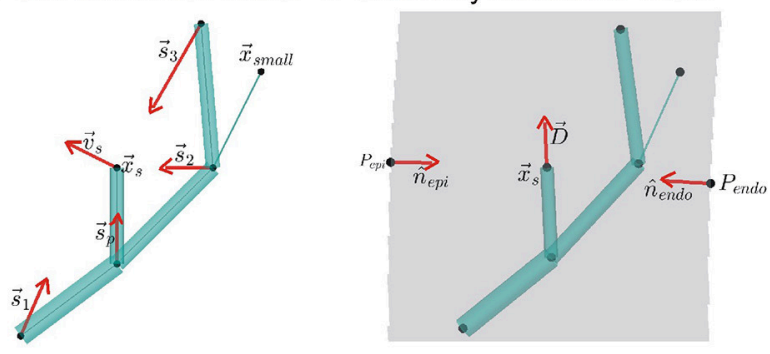

D Assignment of initial coordinates to child nodes

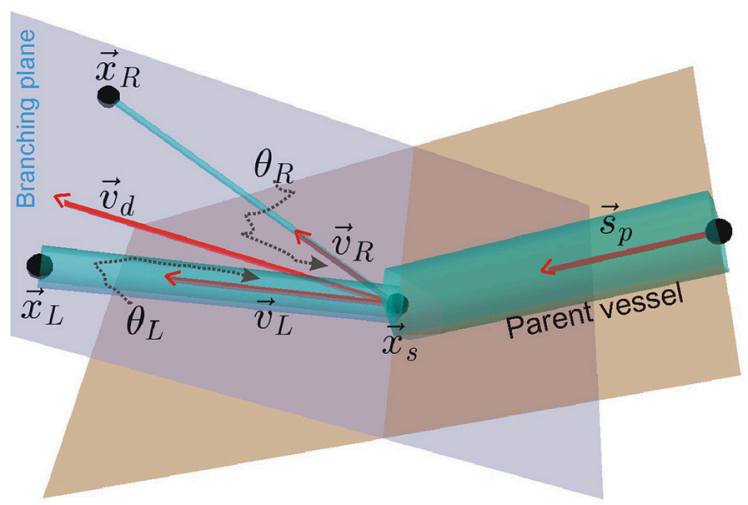

FIGURE 1 | Assignment of coordinates to distal nodes of daughter (or child) segments. (A) Initial branching angles between the left and right daughter segments, $\theta_{L}$ and $\theta_{R}$, we computed using $B F$ and radius ( $r$ ) values of the parent and daughter segments (see Equation 1). The angles are subtended between centrelines (gray lines) of the parent segment and the daughter segments, all of which meet at $\vec{x}_{S}$ the upstream position of the current generating segment. (B) Simplified instance of the dependence of self-avoidance vector, $\vec{v}_{S}$, on supply vectors $\left(\vec{s}_{i}\right)$ from distal nodes of larger segments. In the example network shown, the node $\vec{x}_{\text {small }}$ does not contribute to $\vec{v}_{S}$ because it belongs to a segment that has a smaller radius than both daughter segments being assigned coordinates (daughter segments are shown in A). (C) Same example network of (B) showing the boundary avoidance vector, $\vec{D}$, computed using boundary unit normals $\left(n_{j}\right)$. (D) Using vectors $\vec{s} p$ and $\vec{v}_{d}\left(\vec{v}_{d}\right.$ is a linear combination of $\vec{v}_{s}$ and $\left.\vec{D}\right)$, the initial coordinates of the distal nodes in the branching plane of daughter segments were computed using Equation (8).

where $\vec{s}_{i}=\vec{x}_{c}-\vec{x}_{i}, \vec{x}_{i}$ is upstream position of all segments of same or higher order with coordinates, $\vec{x}_{c}$ is the upstream position of the current generating segment; $d_{i}=\left|\vec{s}_{i}\right|$ is the magnitude of the vector $\vec{s}_{i}$, and $\xi$ is the avoidance exponent with value 2 (Beard and Bassingthwaighte, 2000). 


\section{Boundary avoidance vector}

The boundary avoidance vector permits the assignment of coordinates to child nodes such that they are contained within the predefined ventricle walls. A simple instance of the boundary avoidance vector, $\vec{D}$, is illustrated in Figure 1C. To compute $\vec{D}$, first the analytical equations for the unit normal vectors, $\widehat{n}_{j}$, at each of the $j$ ventricle walls were computed (Section S2, Equation S3). Then, the $\hat{n}_{j}$, distance of upstream position $\vec{x}_{c}$ to boundary $j$ $\left(d_{j}\right)$, and the expected length, $\mathrm{L}$, of the segment being assigned to the daughter segment were used to compute $\vec{D}$ as:

$$
\vec{D}=\sum_{\forall j} n_{j} e^{-d_{j} / 2 L}
$$

\section{Branching plane and assignment of initial coordinates to child nodes}

The self-avoidance and boundary avoidance vectors were combined to generate a combined unit branching plane vector (Figure 1D; Fung et al., 2011; Yang and Wang, 2013; Tamaddon et al., 2016):

$$
\vec{v}_{d}=c_{s} \frac{\vec{v}_{s}}{\left|\vec{v}_{s}\right|}+c_{b} \frac{\vec{D}}{|\vec{D}|}
$$

where $c_{s}=c_{b}=0.5$.

Which was used to generate a unit normal vector $\left(\hat{\mathrm{n}}_{b}\right)$ to the potential branching plane:

$$
\widehat{\mathrm{n}}_{b}=\left(\vec{s}_{p} \times \vec{v}_{d}\right) \times \vec{v}_{d}
$$

Rotation of $\vec{v}_{d}$ around $\hat{\mathrm{n}}_{b}$, by $\theta_{L}$ for left and $\theta_{R}$ for right daughter segments, was performed to compute their respective direction vectors, $v_{L}$ and $v_{R}$. The rotations were implemented using the rotation matrix detailed in Section S5. The initial coordinates of the left and right child nodes, as shown in Figure 1D, were then assigned as follows:

$$
\begin{aligned}
& \vec{x}_{L}=\vec{x}_{c}+L_{L} \vec{v}_{L} \\
& \vec{x}_{R}=\vec{x}_{c}+L_{R} \vec{v}_{R}
\end{aligned}
$$

In case the initially assigned locations (Equation 7) did not lie within ventricle tissue (or at least on the boundary), they were then incrementally moved away from the violating boundary using $\vec{D}$ till $\vec{x}_{L}$ and $\vec{x}_{R}$ fell on or within the tissue. The incremental movement of the nodes was an iterative process and was performed for 200 iterations. Sub-trees that were not assigned positions were then pruned. Upon assignment of positions to all segments and their nodes in the trees, the pressure and BF were computed again using Poiseuille's law.

\section{Generation of ensemble}

As the arterial trees constructed in this study were limited to $\mathrm{SN}$ orders 6-11, any single instance of the geometry was unable to permit calculation of BF heterogeneity (see below). In addition, arterial tree topologies were generated stochastically. Therefore, an ensemble of 540 instances of the human coronary vasculature (consisting of RCA, LAD, and LCX sub-trees) were constructed to allow accurate estimation of $\mathrm{BF}$ in simulation experiments.

\section{Construction of BF Map in 3D, Quantification of Heterogeneity \\ BF map construction}

The model was divided into $1 \mathrm{~mm}^{3}$ (high resolution) or $2 \mathrm{~mm}^{3}$ (low resolution) voxels. BF to a given voxel contained within the ventricular walls was assigned as the sum of BF received through all SN 6 terminals that had coordinates belonging to the volume of that voxel. Such a vowelized distribution of BF was computed for each instance in the ensemble. An average over the complete ensemble was performed to give a BF map. This BF map was used in computing measures for heterogeneity.

\section{BF histograms}

The ranges of $\mathrm{BF}$ values in the vowelized $\mathrm{BF}$ maps were binned, or grouped, into 600 bins for the imaging data, and 100 bins for the modeling data. The numbers of values in each bin were counted and a BF histogram was constructed. The means and standard deviations of the BF histograms were computed.

\section{Probability distribution function of relative BF, fractal dimension}

Probability distribution functions (PDFs) of relative flow were calculated to permit estimation of relative dispersion (Bassingthwaighte et al., 1989). To do so, the vowelized BF values were first normalized as

$$
d_{j}=\sum_{j} \frac{a_{j} / m_{j}}{A / M}
$$

Where $a_{j}$ is the perfusion in the piece, $m_{j}$ is the mass of the piece, $A$ is total perfusion in the $3 \mathrm{D}$ BF map or the imaging data, and $\mathrm{M}$ is the total mass of the ventricles. The $d_{j}$ of Equation 8 is a different quantity from the $d_{j}$ of Equation (4), which is a distance. The masses of 1 voxel in the imaging data, and $1 \mathrm{~mm}^{3}$ voxel in the modeling data were assumed to be 1 . The probability density of each bin was computed to give a probability density function (PDF) over a finite interval histogram. The area under this histogram and its mean were confirmed to be unity, to ensure that this histogram represented a PDF. The standard deviation of this PDF was taken to be the relative dispersion, $\mathrm{RD}$. RD was computed at two resolutions and the lower resolution was taken to be the reference resolution. Fractal dimension, $D$, was computed using these two values of RD using the relationship (Bassingthwaighte et al., 1989):

$$
R D\left(m\left(2 m m^{3}\right)\right)=R D\left(m\left(1 m m^{3}\right)\right)\left(\frac{m\left(2 m m^{3}\right)}{m\left(1 m m^{3}\right)}\right)^{1-D}
$$

where $m$ is the voxel mass at resolution 1 or $2 \mathrm{~mm}^{3}$.

\section{Transmural BF heterogeneity}

To characterize transmural BF heterogeneity, the ventricles were divided into slices of $1 \mathrm{~mm}$ thickness that were equidistant from 
endocardial surfaces. Starting with the structured grid of the BF map, the shortest distance of each $1 \mathrm{~mm}^{3}$ voxels mid-point from the endocardial surfaces was computed. As the surfaces are idealized truncated ellipsoids, a geometric method detailed in Section S4 was implemented to generate distance maps. Using the distances, each voxel's BF was assigned to a $1 \mathrm{~mm}$ thick layer. In particular, the voxels with distances between 1 and $2 \mathrm{~mm}$ from the endocardial surface were considered as the sub-endocardial layer.

\section{Simulation Experiments}

After characterization of the baseline, or control, behavior of the model, simulation experiments were performed. To simulate the effect of adenosine or dialysate cooling, the boundary conditions in terms of inlet pressures were varied from low $(30 \mathrm{mmHg})$ to high $(200 \mathrm{mmHg})$ in steps of $10 \mathrm{mmHg}$, and $\mathrm{BF}$ distribution calculated for each value of inlet pressure. The range of pressures (30-200 $\mathrm{mmHg}$ ) encompassed physiological and pathological aortic pressure. Further, the extreme pressures considered (30 and $200 \mathrm{mmHg}$ ) permitted clear demonstration of the causeeffect relationship in the simulated BF maps. To simulate the effect of dialysis or pre-existing structural conditions, vessels were constricted (stenosis). Stenosis of vessels consisted of either blocking the largest vessels, blocking of vessels that had a particular SN, or other structural characteristics. Finally, the combined effects of altered inlet pressure and blood vessel blocking were simulated.

\section{Numerical Methods}

The topology-geometry algorithms were implemented as a serial computer program using in house developed codes in $\mathrm{C}$ language, and ran on national High Performance Computing Services provided by Compute Canada. Simulation experiments and data analysis were performed using a local cluster. In both cases, the large number of simulations were optimally performed using serial farming job arrays which exploited the GNU parallel LINUX/UNIX utility (Tange, 2011).

\section{RESULTS}

\section{CT Imaging Based BF Heterogeneity in Patient Ventricles}

A representative 3D myocardial BF map which was reconstructed from thoracic CT images is shown in Figure 2, Row 1 and $\mathrm{BF}$ histograms in Figure 2, Row 2. The BF histograms were smoothed using Bezier smoothing and normalized to their respective maximum values to highlight the shifts in peaks. In comparison to control (red lines), adenosine (cyan lines) shifted the histogram peak to higher values. Dialysate cooling (green lines) shifted the histogram's peak in an inconclusive manner. Adenosine with dialysate cooling (blue lines) shifted the peaks to higher values. In case of patients 1 and 2, the histograms are unimodal whereas in case of patient 3, all histograms are bi-modal with one major peak at a high $\mathrm{BF}$ value, along with a secondary peak at a low $B F$ value. The $\mathrm{BF}$ histograms were converted to PDFs of relative flow (see section Methods) (Figure 2, row 3). The standard deviations of these PDFs provided relative dispersions (RDs) at 1 voxel resolution. A similar PDF at a 4 voxel resolution was used to compute fractal dimensions (FDs) (Figure 2, Row 4). In case of all patients, adenosine reduced the 1 voxel $\mathrm{RD}$ and $\mathrm{FD}$ indicating a $\mathrm{BF}$ heterogeneity reducing effect. FD as regards dialysate cooling was either reduced in patients 1 and 2, or increased in case of patient 3 . We noted that the BF histograms and PDFs for patient 3 were bi-modal. The bi-modality of the PDFs was enhanced by application of adenosine and dialysate cooling.

\section{Modeling Results \\ Control in Silico BF Map and Transmural Heterogeneity}

The control in silico BF map and its properties are illustrated in Figure 3. An instance of BF distribution in one of the 541 instances is shown in Figure 3Ai. The average of 541 instances, vowelized at resolution of $1 \mathrm{~mm}^{3}$, was used to represent the control BF map (Figure 3Aii). Histogram of BF, based on the $\mathrm{BF}$ map, shows that the mean $1 \mathrm{~mm}^{3}$ resolution $\mathrm{BF}$ was approximately $3.7 \mathrm{~mm}^{3} / \mathrm{s}$. Histograms at 1 and $2 \mathrm{~mm}^{3}$ resolutions were used to construct the PDF of relative flow (Figure 3Bii shows the $1 \mathrm{~mm}^{3}$ resolution PDF). The FD was found to be 1.18 showing a relatively low $\mathrm{BF}$ heterogeneity in the control BF map. The control in silico BF maps approximate error analysis was performed. FD was computed using an increasing number of instances. The values of FD were found to fit an exponential decay curve exactly (Figure 3Biii). From the fitted curve, it was found that the asymptotic value was 1.14 . The difference between our control BF map and the asymptotic value was $7 \%$, and therefore the error was deemed to be negligible.

Further, the transmural BF heterogeneity was quantified (Figure 4). BF within layers of $1 \mathrm{~mm}$ thickness, computed from the distances of each $1 \mathrm{~mm}^{3}$ voxel from the endocardial surfaces (Figure 4Ai), in the right and left ventricles are shown in Figures $4 \mathrm{Bi}$, Bii respectively. The epicardial layers in the right ventricle received significantly lower $\mathrm{BF}$ than in the left ventricle. The endocardial layers received a significant BF. In both the right and left ventricles, it was found that BF had a minimum in the sub-endocardial layers (Figures $\mathbf{4 B i}$, Bii, gray boxes).

\section{Improved Aortic Pressure Improved Overall BF, Heterogeneity Unaltered}

BF distributions under varying inlet pressure boundary conditions were simulated (Figure 5). The inlet boundary condition, representing aortic pressure, was taken to be 30 , $40,50, \ldots, 200 \mathrm{mmHg}$ and the BF maps calculated at each value of aortic pressure. Figure 5A shows three representative simulated $\mathrm{BF}$ maps at low $(30 \mathrm{mmHg})$, control $(100 \mathrm{mmHg})$, and high (200 $\mathrm{mmHg}$ ) inlet pressures, representing aortic pressures. The BF histograms are shown in Figure 5B. At low pressure (sub-physiological to clearly show the effect), the histogram shows total BF to be low (area under curve of $\mathrm{BF}$ histogram). As inlet pressure was increased, the total BF 


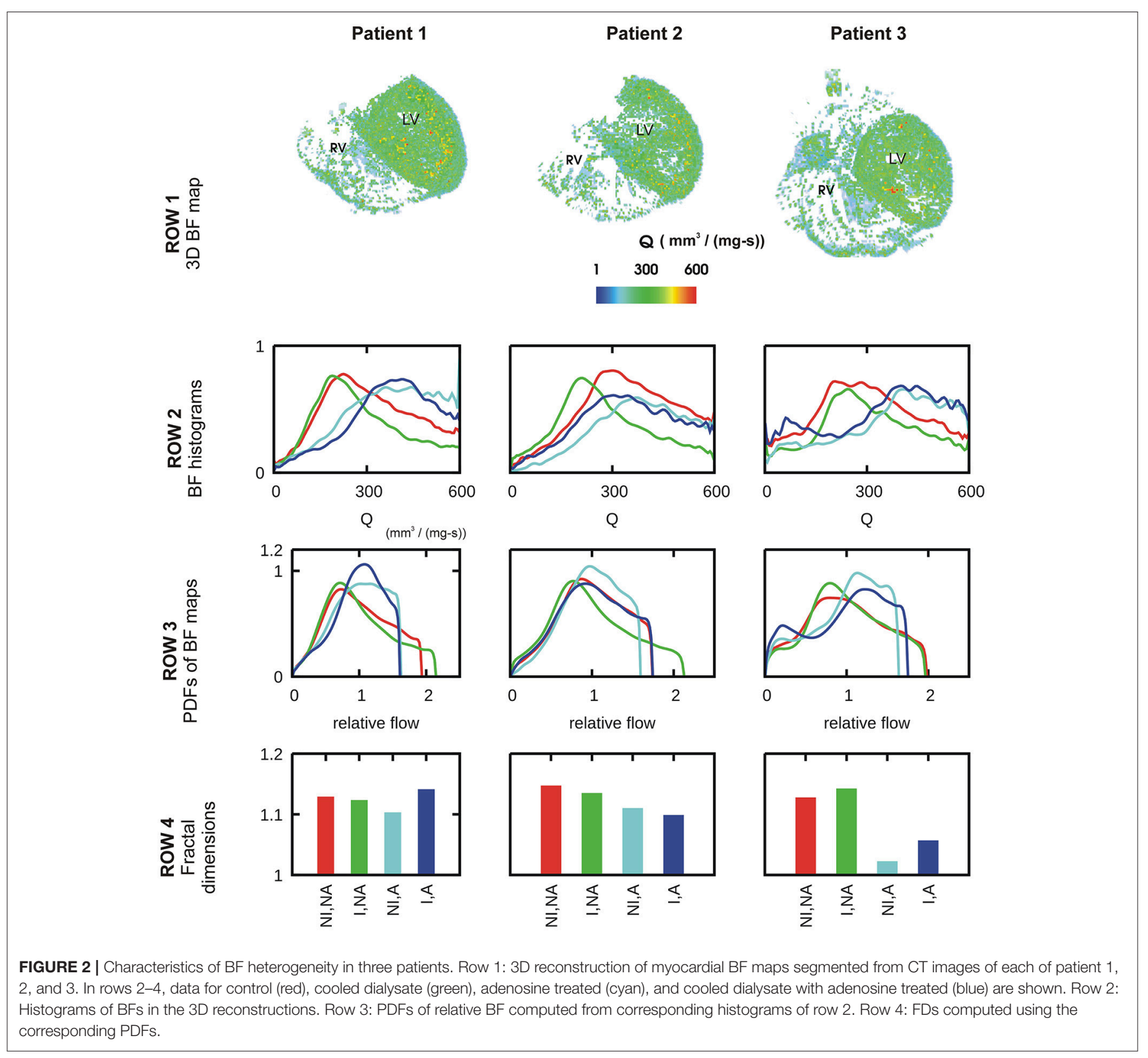

also increased. The linear relationship between total $\mathrm{BF}$ and aortic pressure in Figure 5C shows the constant resistance of the unaltered vascular structure. PDFs of relative perfusion (Figure 5D) confirmed that the RD as well as the consequent FD remained unchanged, due to unaltered underlying coronary structure. The transmural $\mathrm{BF}$ was also unaltered (Figure S3).

\section{Large Artery Blocking (Stenosis) Promotes BF Bi-Modality, Generates Low BF Distal Regions}

The effects of blocking an arbitrarily chosen LAD segment are illustrated in Figure 6. Pressure distributions and BF maps under control ( $r=1.53 \mathrm{~mm}$, Figure 6Ai) and severe stenosis $(r=0.01 \mathrm{~mm}$, Figure 6Aii) illustrate the emergence of low BF distal regions, distal to the severe stenosis. At mild stenosis $(r \geq$ $0.25 \mathrm{~mm}$ ), the PDFs of relative BF were found to be unimodal (Figure 6Bi). In contrast, at severe stenosis $(r<0.25 \mathrm{~mm})$, the histograms became bimodal with two distinct peaks, one each at low and high BF values (Figure 6Bi). As the severity of stenosis was increased, the FD was found to progressively reduce from 1.18 (control) to 1.01 (severe stenosis) (Figure S4). Severe stenosis also reduced the total perfusion by approximately $15 \%$ (Figure S4). However, increased BF heterogeneity is reflected in the increase of RD as the severity of stenosis was increased (Figure 6Bii). The region of $\mathrm{BF}$ distal to the location of stenosis experienced a progressively reduced pressure gradient as shown in Figure 6C. As the stenosis segment was in the LAD and due to the approximate nature of the model (terminals at SN 6), 


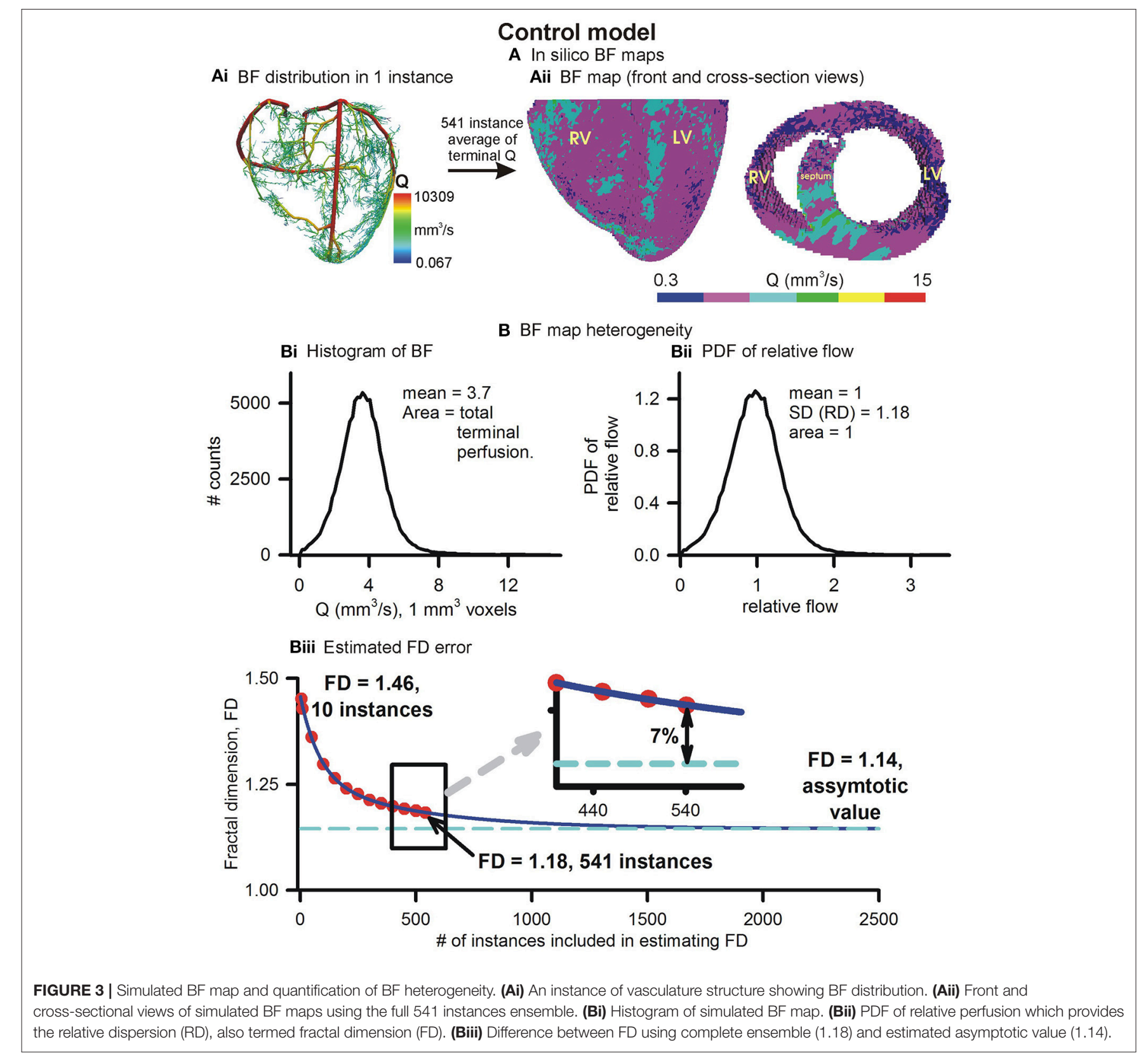

the right ventricle transmural heterogeneity was unaffected (Figure 6Di). However, the amount of BF received by all layers in the left ventricle reduced progressively as severity of stenosis was increased. Further, the transmural heterogeneity was found to reduce as radius of chosen LAD segment was reduced (Figure 6Dii).

\section{Blocking All Large Arteries (Stenosis) Promotes BF Bi-Modality, Blocking Smaller Arterioles Increase BF Heterogeneity}

The roots of arterial sub-trees of a given SN order (SN 6-10) were blocked by $90 \%$ of their control radius to generate BF maps (Figure 7Ai). Blocking of root segments of SN 7-SN 10 sub-trees altered the BF patterns. Blocking of terminals (SN 6) caused an apparent overall reduction of BF. Instances of the corresponding pressure distributions are shown in Figure 7Aii. The BF maps and pressure gradients indicate that the volume of tissue affected is related to the SN order of the arterial sub-tree that was blocked. Relative dispersion of PDFs (Figure 7B) increased $(\mathrm{RD}=1.22$, also see Figure S5) when SN 6 terminals were blocked. The PDF was seen to be uni-modal. Blocking root segments of SN 7 or higher sub-trees also increased relative dispersion, but also gave rise to bi-modal PDFs with peaks at low and high values (Figure 6B). The amplitude of the lower relative flow peak was greater at SN 10 blocking as compared to SN 7 blocking. The total BF when each SN order was blocked is shown in Figure 7C. 


\section{Transmural BF heterogeneity}

\section{Ai. Distance from endo.surface Aii. Transverse section. Aiii. Longitudinal section}
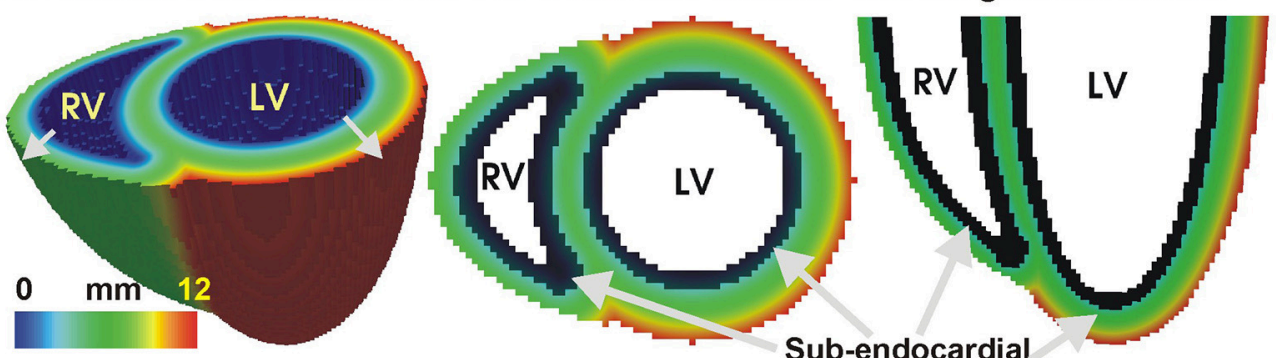

Sub-endocardial

layer defined as

between 1 and $2 \mathrm{~mm}$ from endo surface.

Bi. Right ventricle

Bii. Left ventricle

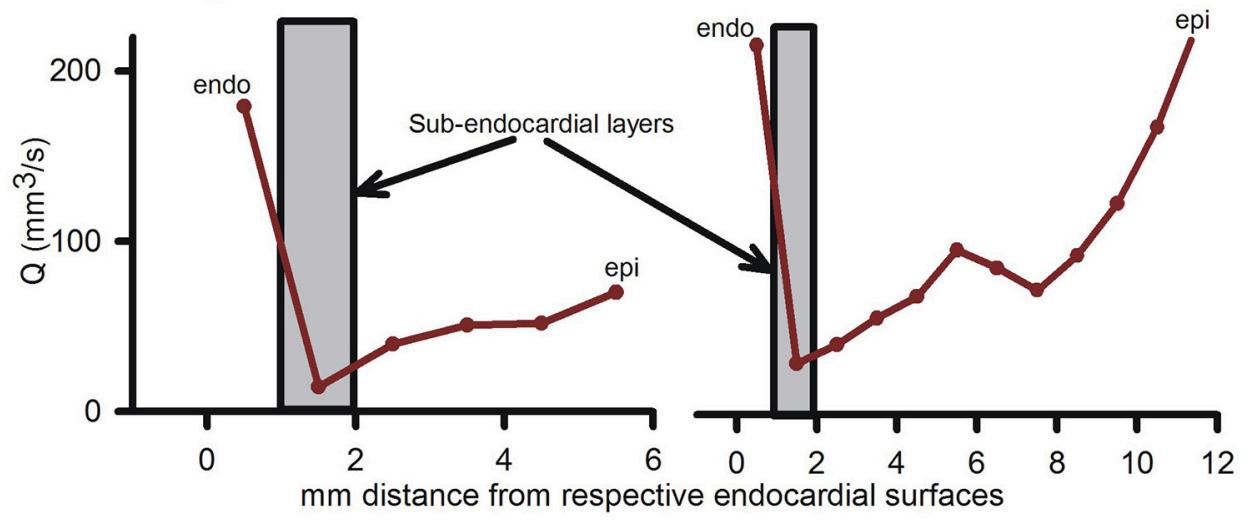

FIGURE 4 | Transmural BF heterogeneity in the simulated BF map. (Ai) Distance of each point in left and right ventricular tissue from respective endocardial surfaces. Increasing distance is shown by gray arrows. (Aii) Cross-section at the base showing distance from endocardial surface. (Aiii) Longitudinal section showing distance from endocardial layer. Black layer in (Aii, Aiii) shows sub-endocardial layer. (Bi) Measured total perfusion in each $1 \mathrm{~mm}$ thick layer of the model right ventricle. (Bii) Measured total perfusion in each $1 \mathrm{~mm}$ thick layer of the model left ventricle. Perfusion in sub-endocardial layer of RV and LV is shown in the gray boxes.

Blocking of the smallest arteries had the most significant effect of reducing total coronary BF. Blocking of SN 7-9 arteries had a relatively less impact. Blocking SN 10 arteries also reduced total coronary BF significantly. Blocking of arteries also affected transmural heterogeneity (Figure 7D). In the right ventricle, blocking SN 7 maximally increased transmural heterogeneity. On the other hand, blocking SN 6, or SN 8-10 reduced transmural heterogeneity. In the left ventricle, blocking SN 6 or SN 10 increased transmural heterogeneity maximally. Blocking of SN 7, 8 , or 9 reduced heterogeneity.

\section{Altered Inlet Perfusion in the Presence of Blocked Arteries}

Simulation of diseased, or adenosine and dialysate cooling therapy induced alteration of aortic pressure in the presence of coronary structural defects are illustrated in Figure 8. Blocking of SN 6 terminals $(70,80$, or $90 \%$ of control radius values) increased the FD (Figure 8A). In contrast, blocking of SN 10 sub-trees reduced the FD. Transmural heterogeneity was unaffected by blocking of SN 6 terminals (Figure 8B). However, as the severity of SN 10 blocking increased, the transmural heterogeneity was found to be increased. Blocking SN 6 vessels has a greater impact on reducing total $\mathrm{BF}$ in comparison to blocking $\mathrm{SN} 10$ vessels (Figure 8C). BF reduced as aortic pressure reduced.

\section{CONCLUSIONS AND DISCUSSION}

The main conclusions of this study are:

a. Clinical imaging can provide information regarding BF alterations in patient ventricles. Total BF can become increased due to pharmacological (adenosine) and therapeutic (dialysate cooling) interventions. Although BF heterogeneity is also potentially affected by the interventions, the information is confounded by underlying vascular structural disease such as large vessel stenosis.

b. Computer modeling indicates that total coronary BF is increased by improved aortic pressure, and also by vasodilation of coronary blood vessels.

c. The causes of BF heterogeneity appear to be multi-fold. Small vessel constriction promoted increase of dispersion but maintained uni-modality of BF histograms. It may be the major cause of $\mathrm{BF}$ heterogeneity. Large vessel constriction promoted bi-modality in BF histograms. Constriction of large 


\section{Improved blood pressure improves coronary perfusion, heterogeniety unaffected}

A Simulated BF maps at low, control, and high aortic pressures.

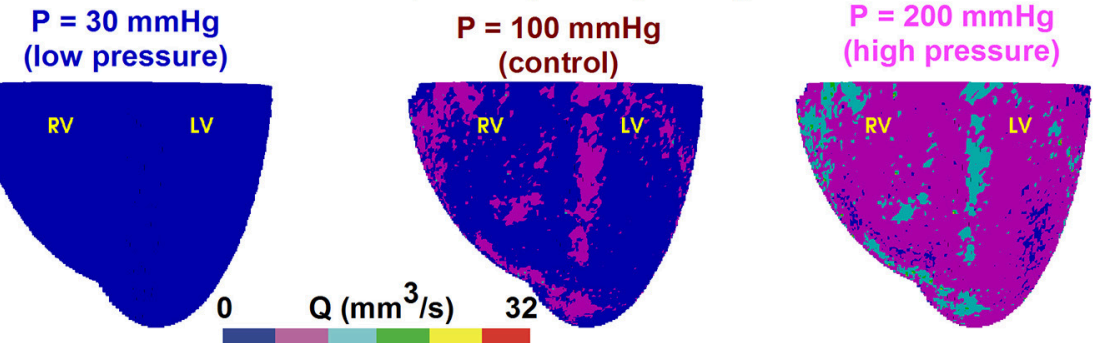

B BF histograms.
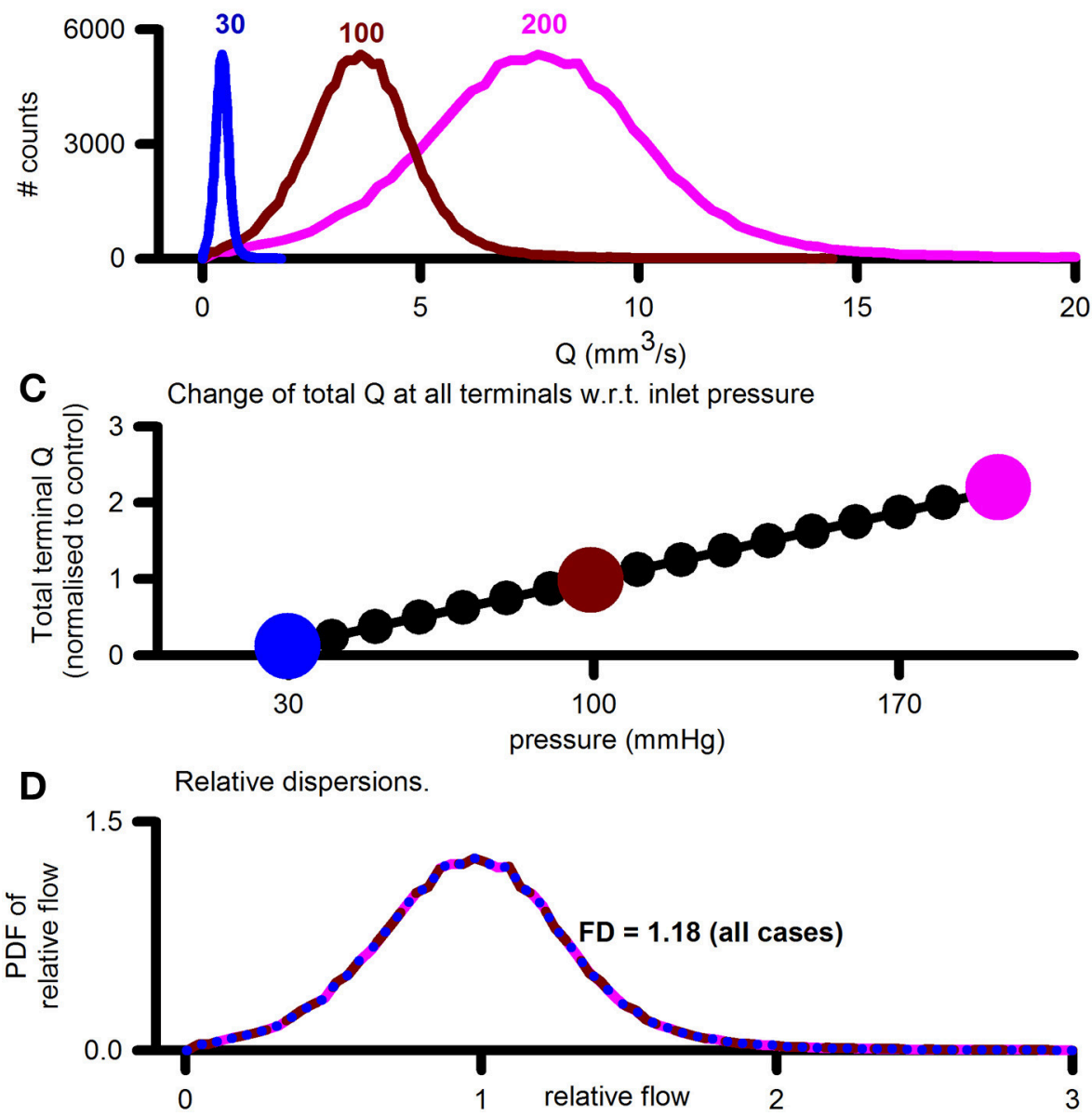

FIGURE 5 | Assessment of the effect of increasing aortic (inlets) pressure. (A) Simulated BF maps at $30 \mathrm{mmHg}$ (left), $100 \mathrm{mmHg}$ (center), and $200 \mathrm{mmHg}$ (right). (B) BF histograms at $30 \mathrm{mmHg}$ (blue), $100 \mathrm{mmHg}$ (red), and $200 \mathrm{mmHg}$ (pink) showing increase of total perfusion with increase of inlet pressure. (C) Total BF at terminals as a function of inlet pressure. Symbols show pressure values where simulations were performed. Large colored circles show the 30,100 , and $200 \mathrm{mmHg}$ values. (D) Color coded PDFs of relative perfusion, which were found to be identical for all pressure values.

vessels had a greater impact on total transmural $\mathrm{BF}$, as well as transmural BF heterogeneity in comparison to constriction of small vessels. The effect of therapy (adenosine or dialysate cooling) may arise from an increase of total BF, caused by an improved aortic pressure.

CT is now an advanced field of imaging that provides a wide spectrum of BF data in the heart (Schindler, 2016; Cademartiri et al., 2017). In this study, the patient hearts were imaged using a rest/stress protocol. It revealed that interventions have a significant effect. The difference between rest/stress also highlighted inherent vascular structural defects in the heart of patient 3 (Figure 2, second row, last column). Our CT protocols are being continually developed to fully exploit available technologies. However, the BF structure-function appears as a 


\section{LAD stenosis at one node alters heterogeneity}

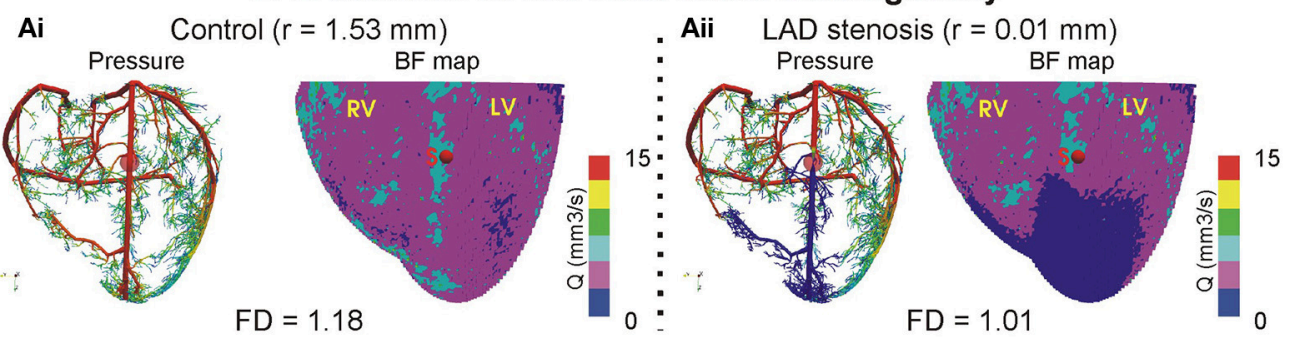

B-D colour legend
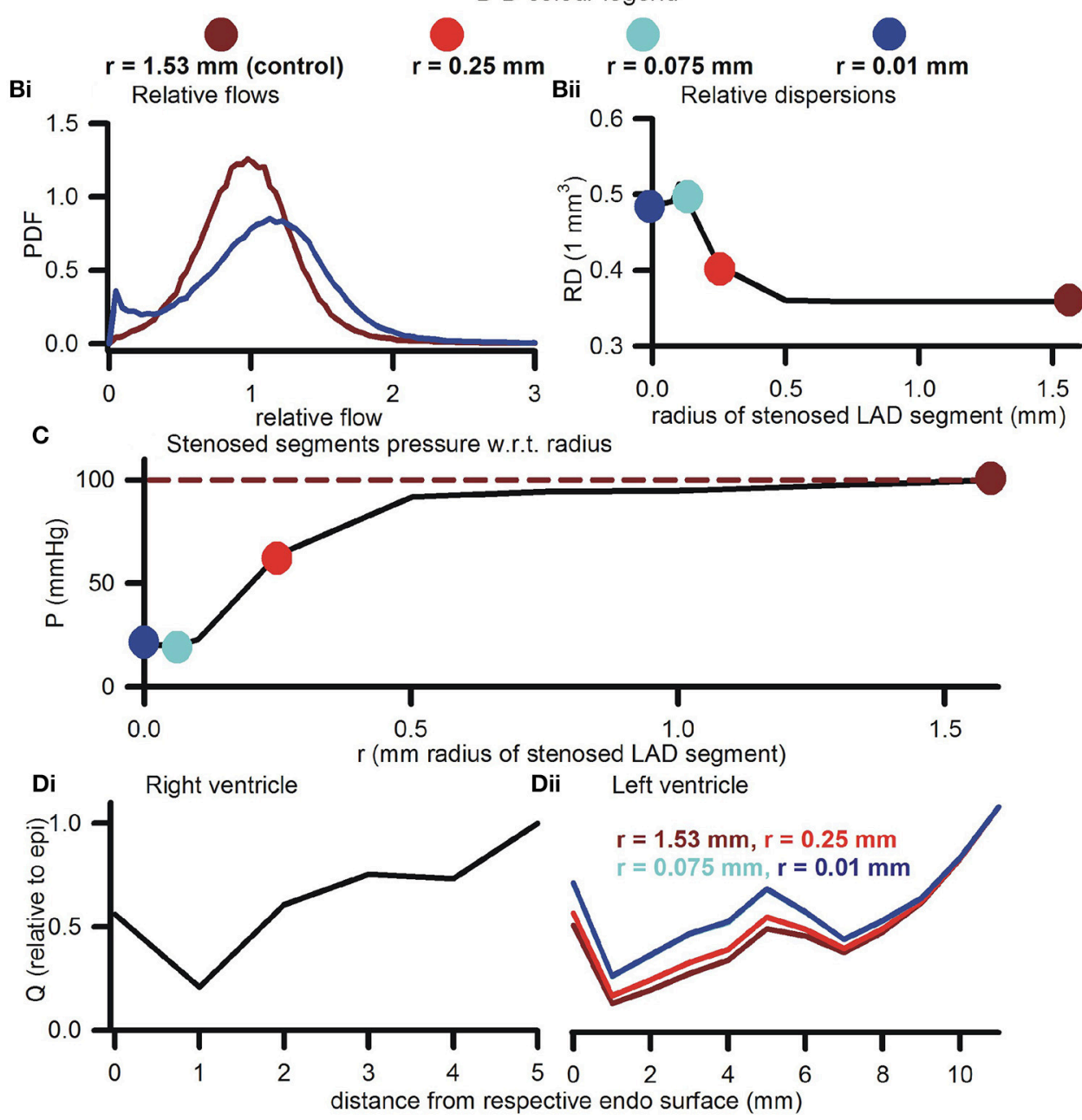

FIGURE 6 | Effect of stenosis in large artery segment. (Ai) Control pressure distribution in vasculature (Left) and BF map (Right). The control radius of the arbitrarily chosen LAD segment was $r=1.53 \mathrm{~mm}$. (Aii) Pressure (Left) and BF map (Right) under severe stenosis at the chosen LAD segment was $r=0.01 \mathrm{~mm}$. In (B-D), the color coding is used to represent segment radii, $r=1.53 \mathrm{~mm}$ (dark red), $r=0.25 \mathrm{~mm}$ (red), $r=0.075 \mathrm{~mm}$ (cyan), and $r=0.01 \mathrm{~mm}$ (blue). (Bi) PDFs of relative perfusion. (Bii) Relationship between RD and stenosed segment radius. (C) Relationship between pressure at stenosed segment and its radius. (Di,Dii) Transmural BF heterogeneity in right (Left) and left (Right) ventricles.

combination of several factors in the imaging, making modeling investigation necessary.

Based on experimental measurements, our model incorporates morphometry data that gave realistic topologies of the coronary vasculature. The optimal assignment of $3 \mathrm{D}$ positions to the arterial tree nodes was performed using space filling algorithm (Beard and Bassingthwaighte, 2000). Accounting for limitations, an ensemble of instances was generated whose average hemodynamic properties are presented. The presented models $\mathrm{BF}$ heterogeneity ( $\mathrm{FD}=1.14)$ is in 

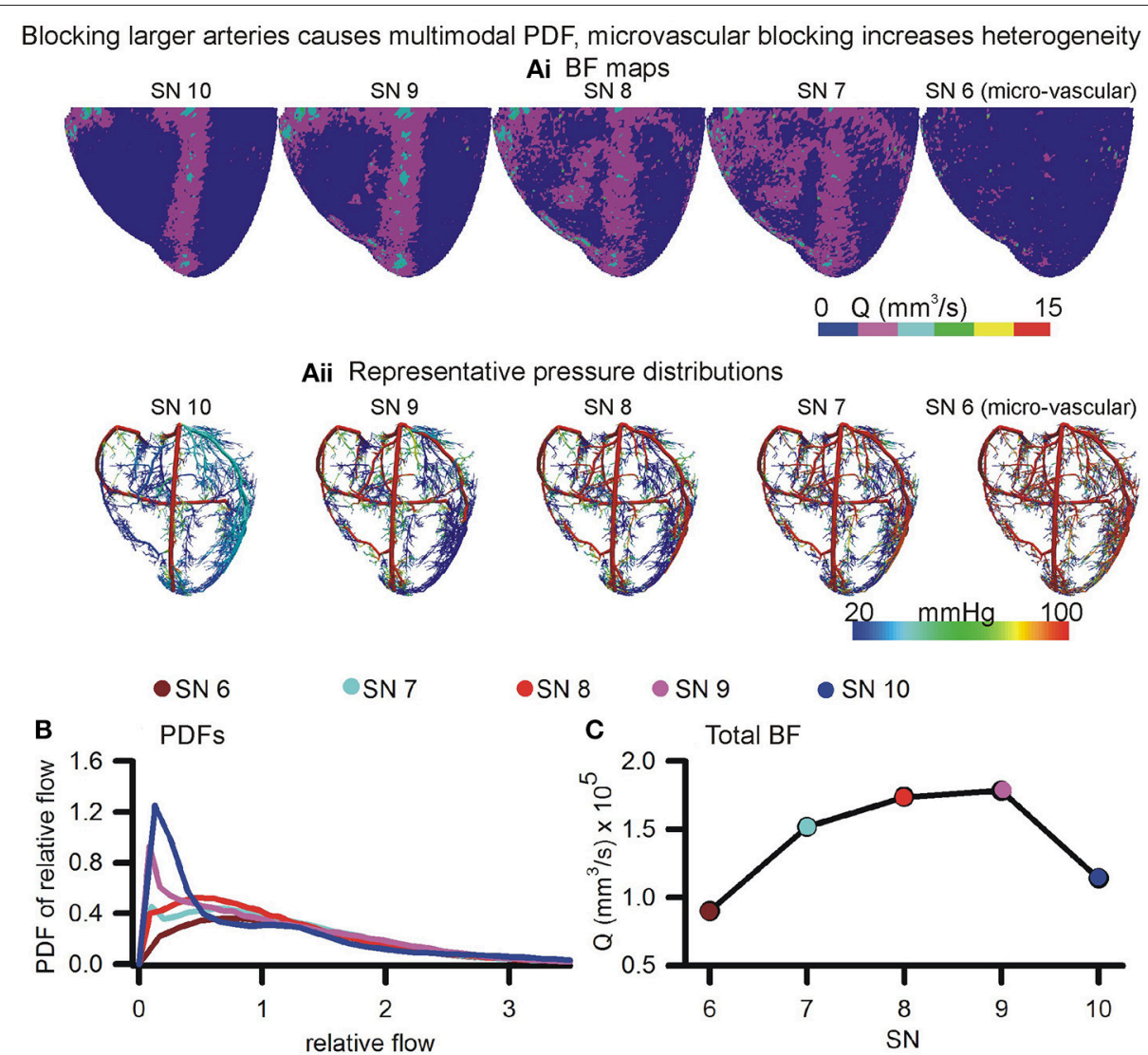

Aii Representative pressure distributions
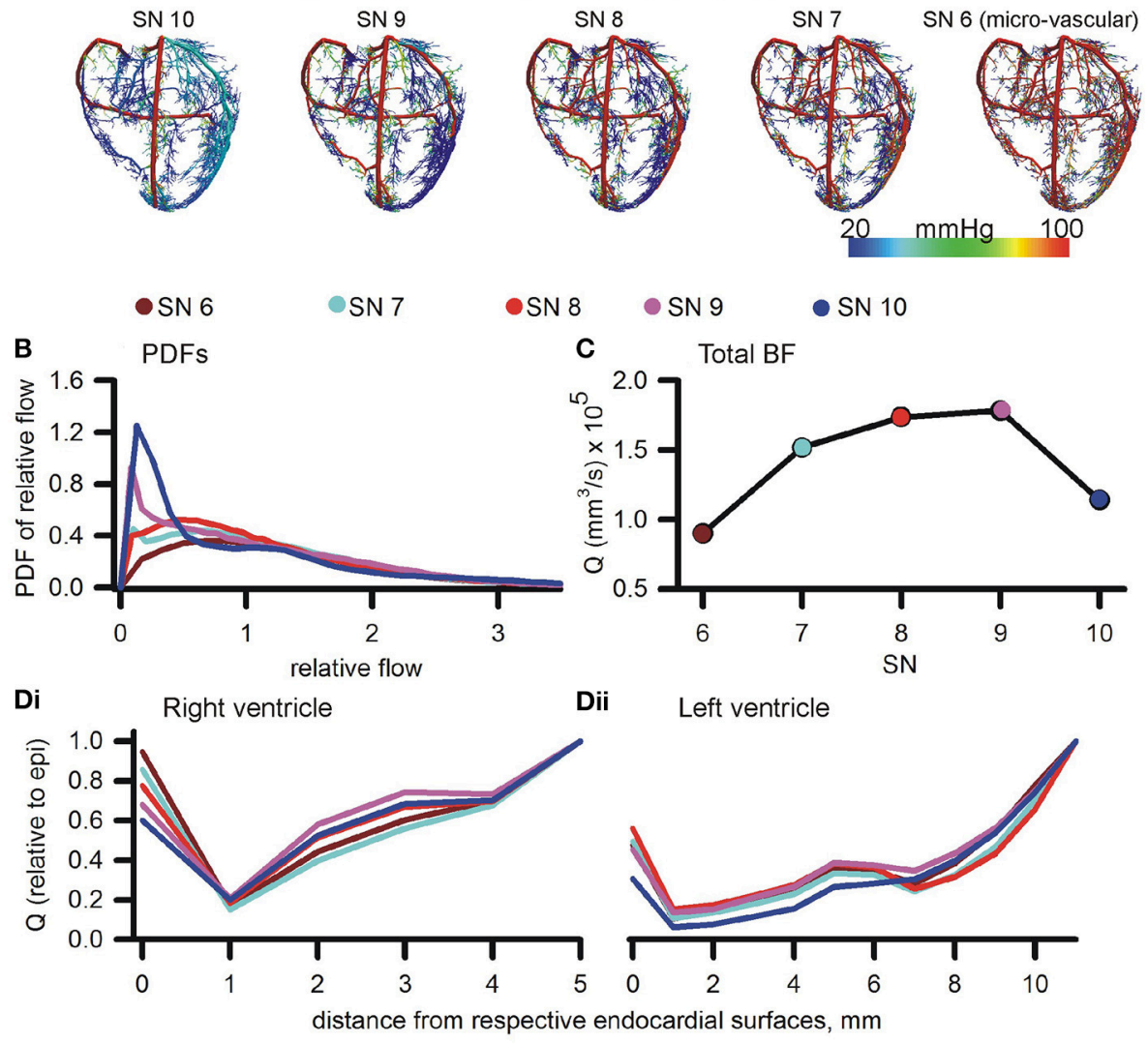

FIGURE 7 | Effects of blocking arteries at given order on perfusion and heterogeneity. (Ai,Aii) BF maps (Ai) and pressure distributions (Aii) when arteries of various orders were blocked. (B) PDFs of BF maps. (C) Total BF at terminals when that order arteries were blocked. (Di,Dii) Epi-endo heterogeneity alterations under blocking of various orders of arteries.

agreement with FD of 1.2 observed by VanBavel and Spaan in microcirculation SN 2-8 networks (VanBavel and Spaan, 1992). Others have used optimized models, where a spectrum of cost functions such as single arterial volume (Karch et al., 1999; Schreiner et al., 2006), or the combination of metabolic cost, volume, power (Kaimovitz et al., 2005; Keelan et al., 2016) have been optimized. Notwithstanding the relatively simple optimization used in this study, Figure 4 shows that the BF is distributed throughout most of the myocardium almost uniformly $(\mathrm{FD}=1.18)$. Our model has characteristic transmural $\mathrm{BF}$ heterogeneity. Whereas total path lengths of vessels were constrained, the combination of transmural path lengths and the space filling-boundary avoidance algorithm may explain model behavior. It is known that the myocardium is heterogeneously perfused transmurally (Huo et al., 2009). We therefore computed to alteration of our models transmural heterogeneity as an approximation, and we bear in mind that further development is required.

In this study, multi-modality in BF histograms due to large vessel severe stenosis was observed in the model, which agrees with the imaging data. However, single segment stenosis, or stenosis of high order segments, may be accompanied by an autoregulatory response. Although overall $\mathrm{BF}$ became multi-modal, the FD (computed using the same methods as well as binning) was observed to be reduced. In contrast, the modeling study by Meier et al. (2004) which included autoregulation showed 


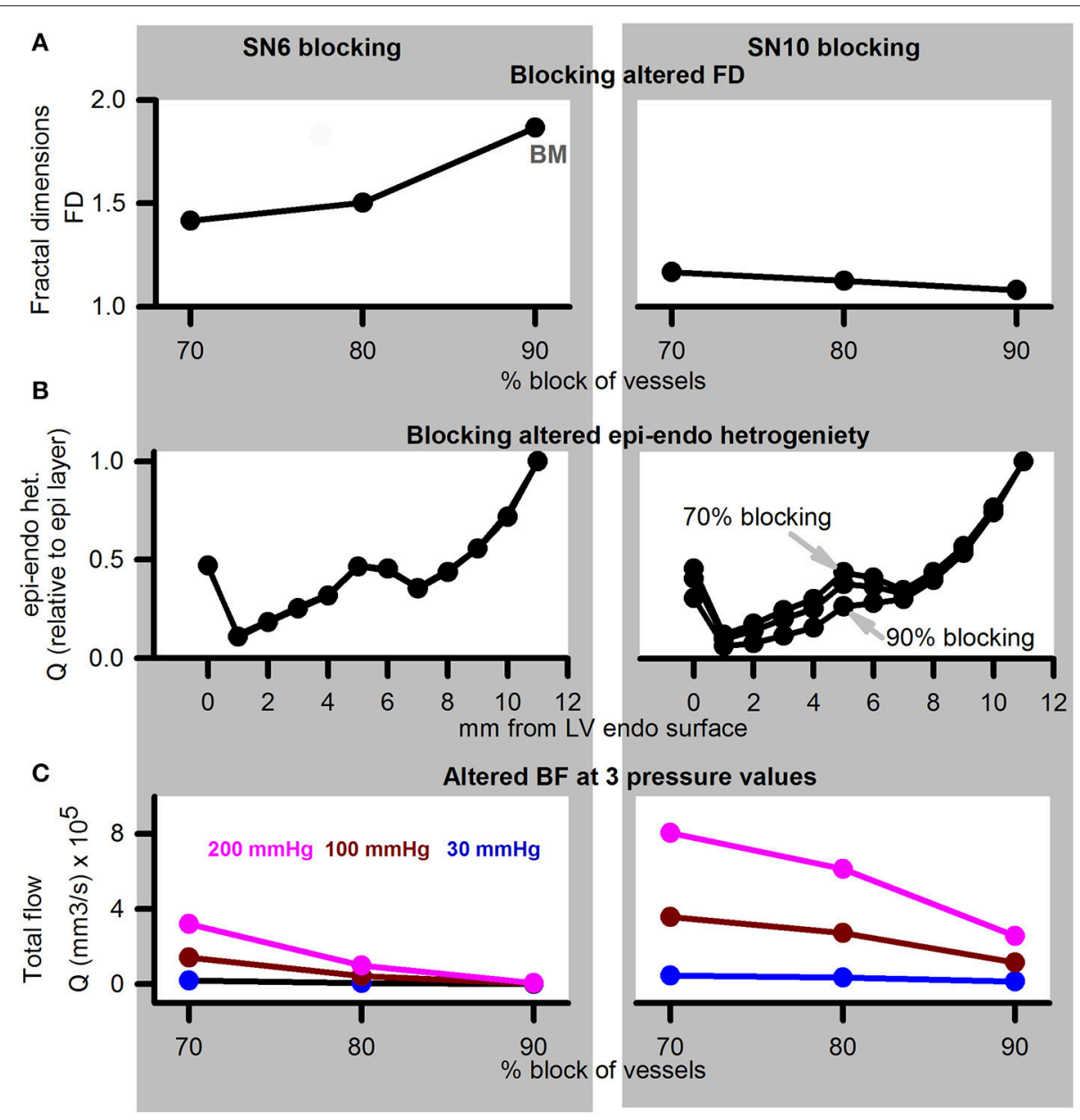

FIGURE 8 | Simulation of disease (reduced pressure) or adenosine and dialysate cooling (therapy) in the presence of structural defects. Left column shows data for SN 6 blocking, while right column shows data for SN 10 blocking. (A) FD when vessels were blocked by 70, 80, or 90\% of their control values. (B) Transmural BF heterogeneity. (C) Total BF when vessels were blocked by 70, 80, or 90\% when applied inlet pressure was $200 \mathrm{mmHg}$ (pink), $100 \mathrm{mmHg}$ (dark red), or $30 \mathrm{mmHg}$ (blue).

that FD remained unchanged under stenosis. Remarkably, the inclusion of autoregulation in the above study maintained uni-modality of BF histograms, while increasing RD when low aortic pressure was applied.

The present model was constructed using a limited topology (SN 6-SN 11). It was also optimized using relatively straightforward space filling and boundary avoidance conditions. Nevertheless, transmural BF heterogeneity was observed, and it was affected by structural alterations. Specifically, blocking the larger ( $\mathrm{SN} 7$ or 8 ) vessels increased transmural heterogeneity more than blocking of the microvasculature (SN 6). Our finding is in line with that of Algranati et al. (2011) who have identified sub-endocardial compliance as a cause of BF redistribution. In the above study, they have also identified the contractile factors that may contribute to the sub-endocardial heterogeneity.

Although there have been significant advances in the experimental, theoretical, and imaging literature, we believe that this is one of a few studies that applies coronary vasculature models to indicatively explain clinical imaging observations. Previously developed models have generated 4D XCAT Phantoms (Fung et al., 2011), or assist the clinician in identifying clinical defects (Fung et al., 2010). Further development of the presented model, at multi-scale and at multiphysics levels, will incorporate Cardiac Physiome models in a pre-clinical assessment tool to assist our clinical research. The model, in conjunction with detailed imaging (see Jogiya et al., 2014, as well as Figure 1), will expedite the assessment of patient BF status and consequently overall health assessment. The model is also capable of permitting investigation of specific artery bifurcation properties under health and disease conditions (Auricchio et al., 2014). Investigation of properties such as electrical wave propagation and their interaction with vascular structure (Bishop et al., 2010) can be further investigated using the presented model. Extension of the model will incorporate the multi-scale nature of delivery of oxygen to myocardial tissue (Goldman et al., 2017; Mason McClatchey et al., 2017). 


\section{LIMITATIONS}

\section{Imaging Limitations}

Although a large amount of information is available in our clinical images, certain limitations remain. Firstly, only the diastolic phase was collected. During left ventricle's diastole, the right ventricle is still moving and therefore cannot be imaged. Using a 4D scanning protocol may alleviate this limitation in future studies that can capture right ventricle signals.

Another limitation is that of resolution. Due to the toxicity of the contrast agent and other factors, it is difficult to image the patient for longer durations. However, that results in significantly less number of slices and low 3D resolution. At the acquired resolution, it may not be possible to gain insights into the precapillary arteriole BF distributions. Apart from CT, other imaging modalities may be available for assessment of arterial defects (Gharib et al., 2008). The issues regarding toxicity of CT contrast agents may be reduced using magnetic resonance based imaging (Jogiya et al., 2014).

\section{Modeling Limitations}

Our model is limited to SN 6 (arterioles) to SN 11 (main coronary arteries) arterial segments. The ensemble of coronary trees generated in this work is based on the morphometric data by the Kassab group. However, the optimization algorithm in the uneven geometry of the heart is a compute intensive task and it was not possible to generate optimized structures within a short $(48 \mathrm{~h})$ duration. It is known that assigning spatial locations to the vasculature nodes is a large optimization problem where fractal models based on morphometry need to be combined with theoretical optimization methods (Schreiner et al., 2006). In future work, we will consider inclusion on further biophysical principles that permit use of cost functions to compute optimal locations of arteriole terminals (Zamir and Phipps, 1988; Kaimovitz et al., 2010; Keelan et al., 2016).

An important simplification of our model is that diameter asymmetry has been ignored. In our model, elements of the same order have a uniform radius. In addition, the bifurcations are assigned randomly where the daughter element radii are assigned according to morphometry rather than according to BF symmetry. Blood vessel diameter asymmetry has been observed in casting data (Huo et al., 2009) and shown to affect BF distributions significantly (Kaimovitz et al., 2008). Bifurcation asymmetry is known to promote increased RD (Sriram et al., 2014) and may be an important factor that contributes to BF heterogeneity in the critically ill groups of patients (Frisbee et al., 2016). It has been quantified in past studies (VanBavel and Spaan, 1992; Dankelman et al., 2007) and will be included in future versions of our model.

Both pulsatile BF (Huo and Kassab, 2006) and viscosity variability in micro-vessels (Pries et al., 1996; Pries and Secomb, 2009) have been ignored for simplicity in this study. As a first step toward constructing our model, the present study assumed steady state BF in line with the literature (Karch et al., 1999; Beard and Bassingthwaighte, 2000; Keelan et al., 2016), which includes studies that quantify BF heterogeneity (Schreiner et al., 2003). The coronary artery diameter $(3.2 \mathrm{~mm})$ is 10 times less than that of the ascending aorta during diastole ( $\sim 32 \mathrm{~mm}$ ) (de Heer et al., 2011), thus partially justifying the present study's steady state BF assumption. It should also be appreciated that this study's CT imaging data were obtained during the diastole when pressure changes may be small. The overall qualitative results of this study regarding $3 \mathrm{D}$ structure based distribution of blood in the ventricles may remain unaffected with the inclusion of pulsatile BF, but also limit the applicability of the model. The pulsatile nature of blood flow in the coronary arteries stems from the pulsating pressure boundary conditions at the aorta, as well as the effects of myocardial motion-contraction. A number of previous theoretical and experiment-modeling studies (Womersley, 1955, 1958; Duan and Zamir, 1995; Huo and Kassab, 2006, 2007) may be adapted to incorporate pulsatile boundary conditions in the present model. The adaptation of previous knowledge entails significant model development and is out of scope of this study. In future studies, the inclusion of the time varying boundary conditions will permit application of model to address clinically relevant challenges such as calculation of fractional flow reserve (Yong et al., 2017) and vascular surgery assessment (Kouhi et al., 2008).

Generation of geometry is based on deterministic space filling, whereas optimization of energy expenditure or some measure is expected to give improved distribution in the future. In the future, we aim to develop a $4 \mathrm{D} \mathrm{XCT}$ phantom that will permit a more in depth patient specific BF assessment along with other parameters (Fung et al., 2011).

The lack of autoregulation is a significant simplification in our study. The inclusion of autoregulation may affect results presented in this study (Meier et al., 2004). It will also permit testing of further factors that affect local and global flow, as shown recently by Namani et al. (2018).

Apart from structural changes, $\mathrm{BF}$ is also affected by several other factors, where metabolic demand of tissue and protein expression heterogeneity are prominent (Stoll et al., 2008). Autoregulation, as well as other patho-physiological factors will be incorporated into the model in future work.

\section{CLINICAL SIGNIFICANCE OF THE STUDY}

In this study, imaging data acquired from patients was analyzed. Whereas it is probably true that dialysis causes vascular dysfunction by affecting the micro-vasculature, this study's imaging observation combined with the modeling results, indicates that large vessel dysfunction may significantly affect patient's coronary perfusion.

In the process of quantifying BF heterogeneity, we have now developed algorithms that compute simple yet informative measures of BF heterogeneity. Such a tool will provide rapid assessment of whether imaging data reflect the effectiveness of therapy.

An important development during this study was the implementation of a method to generate organ level vascular structure. When combined with micro-vasculature, the vasculature model is being developed to become a pre-clinical trial in silico indicative outcome assessment tool. 


\section{AUTHOR CONTRIBUTIONS}

SK, DG, and CM designed the study in consultation with CE. AS, and T-YL provided the texture analyzed CT data and related text. SK and FS segmented the hearts. SK developed the codes and performed the simulation experiments, acquired, curated, and analyzed the data. DG and CM provided expert comments. CE provided expert insights into clinical-modeling data interpretation. SK wrote the first draft. AS provided the draft of the imaging data analysis. All authors wrote and approved the final manuscript.

\section{REFERENCES}

Alarcón, T., Byrne, H. M., and Maini, P. K. (2005). A design principle for vascular beds: the effects of complex blood rheology. Microvasc. Res. 69, 156-172. doi: 10.1016/j.mvr.2005.02.002

Algranati, D., Kassab, G. S., and Lanir, Y. (2011). Why is the subendocardium more vulnerable to ischemia? A new paradigm. Am. J. Physiol. Heart Circ. Physiol. 300, H1090-1100. doi: 10.1152/ajpheart.00473.2010

Auricchio, F., Conti, M., Ferrazzano, C., and Sgueglia, G. A. (2014). A simple framework to generate 3D patient-specific model of coronary artery bifurcation from single-plane angiographic images. Comput. Biol. Med. 44, 97-109. doi: 10.1016/j.compbiomed.2013.10.027

Bassingthwaighte, J. B., King, R. B., and Roger, S. A. (1989). Fractal nature of regional myocardial blood flow heterogeneity. Circ. Res. 65, 578-590. doi: 10.1161/01.RES.65.3.578

Beard, D. A., and Bassingthwaighte, J. B. (2000). The fractal nature of myocardial blood flow emerges from a whole-organ model of arterial network. J. Vasc. Res. 37, 282-296. doi: 10.1159/000025742

Bishop, M. J., Boyle, P. M., Plank, G., Welsh, D. G., and Vigmond, E. J. (2010). Modeling the role of the coronary vasculature during external field stimulation. IEEE Trans. Biomed. Eng. 57, 2335-2345. doi: 10.1109/TBME.2010.20 51227

Breidthardt, T., Burton, J. O., Odudu, A., Eldehni, M. T., Jefferies, H. J., and McIntyre, C. W. (2012). Troponin T for the detection of dialysis-induced myocardial stunning in hemodialysis patients. Clin. J. Am. Soc. Nephrol. 7, 1285-1292. doi: 10.2215/CJN.00460112

Burton, J. O., Jefferies, H. J., Selby, N. M., and McIntyre, C. W. (2009). Hemodialysis-induced repetitive myocardial injury results in global and segmental reduction in systolic cardiac function. Clin. J. Am. Soc. Nephrol. 4, 1925-1931. doi: 10.2215/CJN.04470709

Cademartiri, F., Seitun, S., Clemente, A., La Grutta, L., Toia, P., Runza, G., et al. (2017). Myocardial blood flow quantification for evaluation of coronary artery disease by computed tomography. Cardiovasc. Diagn. Ther. 7, 129-150. doi: $10.21037 /$ cdt.2017.03.22

Collins, A. J., Foley, R. N., Gilbertson, D. T., and Chen, S. C. (2015). United States Renal Data System public health surveillance of chronic kidney disease and end-stage renal disease. Kid. Int. Suppl. 5, 2-7. doi: 10.1038/kisup.2015.2

Dankelman, J., Cornelissen, A. J., Lagro, J., Vanbavel, E., and Spaan, J. A. (2007). Relation between branching patterns and perfusion in stochastic generated coronary arterial trees. Med. Biol. Eng. Comput. 45, 25-34. doi: 10.1007/s11517-006-0139-9

Dasselaar, J. J., Slart, R. H., Knip, M., Pruim, J., Tio, R. A., McIntyre, C. W., et al. (2009). Haemodialysis is associated with a pronounced fall in myocardial perfusion. Nephrol. Dial. Transplant 24, 604-610. doi: 10.1093/ndt/gfn501

de Heer, L. M., Budde, R. P., Mali, W. P., de Vos, A. M., van Herwerden, L. A., and Kluin, J. (2011). Aortic root dimension changes during systole and diastole: evaluation with ECG-gated multidetector row computed tomography. Int. J. Cardiovasc. Imaging 27, 1195-1204. doi: 10.1007/s10554-011-9838-x

Dodge, J. T. Jr., Brown, B. G., Bolson, E. L., and Dodge, H. T. (1992). Lumen diameter of normal human coronary arteries. Influence of age, sex, anatomic variation, and left ventricular hypertrophy or dilation. Circulation 86, 232-246. doi: 10.1161/01.CIR.86.1.232

\section{ACKNOWLEDGMENTS}

We thank LHSC IT, Compute Canada, SHARCNet for IT and HPC resources. This study was supported by Heart and Stroke grant (G-17-0018311, PI: CM).

\section{SUPPLEMENTARY MATERIAL}

The Supplementary Material for this article can be found online at: https://www.frontiersin.org/articles/10.3389/fphys. 2018.00511/full\#supplementary-material

Duan, B., and Zamir, M. (1995). Pressure peaking in pulsatile flow through arterial tree structures. Ann. Biomed. Eng. 23, 794-803. doi: 10.1007/BF02584478

Frisbee, J. C., Goodwill, A. G., Frisbee, S. J., Butcher, J. T., Wu, F., and Chantler, P. D. (2016). Microvascular perfusion heterogeneity contributes to peripheral vascular disease in metabolic syndrome. J. Physiol. (Lond). 594, 2233-2243. doi: 10.1113/jphysiol.2014.285247

Fung, G. S., Segars, W. P., Gullberg, G. T., and Tsui, B. M. (2011). Development of a model of the coronary arterial tree for the 4D XCAT phantom. Phys. Med. Biol. 56, 5651-5663. doi: 10.1088/0031-9155/56/17/012

Fung, G. S., Segars, W. P., Lee, T. S., Higuchi, T., Veress, A. I., Gullberg, G. T., et al. (2010). Realistic simulation of regional myocardial perfusion defects for cardiac SPECT studies. IEEE Nucl. Sci. Symp. Conf. Rec. 2010, 3061-3064. doi: 10.1109/NSSMIC.2010.5874362

Gharib, A. M., Ho, V. B., Rosing, D. R., Herzka, D. A., Stuber, M., Arai, A. E., et al. (2008). Coronary artery anomalies and variants: technical feasibility of assessment with coronary MR angiography at 3 T. Radiology 247, 220-227. doi: 10.1148/radiol.2471070274

Göktepe, S., and Kuhl, E. (2010). Electromechanics of the heart: a unified approach to the strongly coupled excitation-contraction problem. Comput. Mech. 45, 227-243. doi: 10.1007/s00466-009-0434-Z

Goldman, D., Zahra Farid, K. A. L., Jefferson, C., Frisbee, and D. N. J. (2017). "The microcirculation physiome: an approach using arteriolar-venular networks reconstructed from in vivo data," in Cardiac Physiome Society Meeting (Toronto, ON).

Hacking, W. J., VanBavel, E., and Spaan, J. A. (1996). Shear stress is not sufficient to control growth of vascular networks: a model study. Am. J. Physiol. 270, H364-H375. doi: 10.1152/ajpheart.1996.270.1.H364

Huo, Y., Kaimovitz, B., Lanir, Y., Wischgoll, T., Hoffman, J. I., and Kassab, G. S. (2009). Biophysical model of the spatial heterogeneity of myocardial flow. Biophys. J. 96, 4035-4043. doi: 10.1016/j.bpj.2009.02.047

Huo, Y., and Kassab, G. S. (2006). Pulsatile blood flow in the entire coronary arterial tree: theory and experiment. Am. J. Physiol. Heart Circ. Physiol. 291, H1074-H1087. doi: 10.1152/ajpheart.00200.2006

Huo, Y., and Kassab, G. S. (2007). A hybrid one-dimensional/Womersley model of pulsatile blood flow in the entire coronary arterial tree. Am. J. Physiol. Heart Circ. Physiol. 292, H2623-H2633. doi: 10.1152/ajpheart.00987.2006

Jefferies, H. J., Virk, B., Schiller, B., Moran, J., and McIntyre, C. W. (2011). Frequent hemodialysis schedules are associated with reduced levels of dialysis-induced cardiac injury (myocardial stunning). Clin. J. Am. Soc. Nephrol. 6, 1326-1332. doi: 10.2215/CJN.05200610

Jogiya, R., Morton, G., De Silva, K., Reyes, E., Hachamovitch, R., Kozerke, S., et al. (2014). Ischemic burden by 3-dimensional myocardial perfusion cardiovascular magnetic resonance: comparison with myocardial perfusion scintigraphy. Circ. Cardiovasc. Imaging 7, 647-654. doi: 10.1161/CIRCIMAGING.113.001620

Kaimovitz, B., Huo, Y., Lanir, Y., and Kassab, G. S. (2008). Diameter asymmetry of porcine coronary arterial trees: structural and functional implications. Am. J. Physiol. Heart Circ. Physiol. 294, H714-H723. doi: 10.1152/ajpheart.00818.2007

Kaimovitz, B., Lanir, Y., and Kassab, G. S. (2005). Large-scale 3-D geometric reconstruction of the porcine coronary arterial vasculature based on detailed anatomical data. Ann. Biomed. Eng. 33, 1517-1535. doi: 10.1007/s10439-005-7544-3 
Kaimovitz, B., Lanir, Y., and Kassab, G. S. (2010). A full 3-D reconstruction of the entire porcine coronary vasculature. Am. J. Physiol. Heart Circ. Physiol. 299, H1064-H1076. doi: 10.1152/ajpheart.00151.2010

Karch, R., Neumann, F., Neumann, M., and Schreiner, W. (1999). A three-dimensional model for arterial tree representation, generated by constrained constructive optimization. Comput. Biol. Med. 29, 19-38. doi: 10.1016/S0010-4825(98)00045-6

Kassab, G. S., Berkley, J., and Fung, Y. C. (1997a). Analysis of pig's coronary arterial blood flow with detailed anatomical data. Ann. Biomed. Eng. 25, 204-217. doi: 10.1007/BF02738551

Kassab, G. S., Pallencaoe, E., Schatz, A., and Fung, Y. C. (1997b). Longitudinal position matrix of the pig coronary vasculature and its hemodynamic implications. Am. J. Physiol. 273, H2832-H2842. doi: 10.1152/ajpheart.1997.273.6.H2832

Kassab, G. S., Rider, C. A., Tang, N. J., and Fung, Y. C. (1993). Morphometry of pig coronary arterial trees. Am. J. Physiol. 265, H350-H365. doi: 10.1152/ajpheart.1993.265.1.H350

Keelan, J., Chung, E. M., and Hague, J. P. (2016). Simulated annealing approach to vascular structure with application to the coronary arteries. R. Soc. Open Sci. 3:150431. doi: 10.1098/rsos.150431

Kouhi, E., Morsi, Y. S., and Masood, S. H. (2008). Haemodynamic analysis of coronary artery bypass grafting in a non-linear deformable artery and Newtonian pulsatile blood flow. Proc. Inst. Mech. Eng. H 222, 1273-1287. doi: 10.1243/09544119JEIM459

Mason McClatchey, P., Wu, F., Olfert, I. M., Ellis, C. G., Goldman, D., Reusch, J. E., et al. (2017). Impaired tissue oxygenation in metabolic syndrome requires increased microvascular perfusion heterogeneity. J. Cardiovasc. Transl. Res. 10, 69-81. doi: 10.1007/s12265-017-9732-6

McIntyre, C. W. (2010). Haemodialysis-induced myocardial stunning in chronic kidney disease - a new aspect of cardiovascular disease. Blood Purif. 29, 105-110. doi: 10.1159/000245634

McIntyre, C. W., and Odudu, A. (2014). Hemodialysis-associated cardiomyopathy: a newly defined disease entity. Semin. Dial. 27, 87-97. doi: 10.1111/sdi. 12197

McIntyre, N. J., Fluck, R. J., McIntyre, C. W., Fakis, A., and Taal, M. W. (2013). Determinants of arterial stiffness in chronic kidney disease stage 3. PLoS ONE 8:e55444. doi: 10.1371/journal.pone.0055444

Meier, J., Kleen, M., and Messmer, K. (2004). A computer model of fractal myocardial perfusion heterogeneity to elucidate mechanisms of changes in critical coronary stenosis and hypotension. Bull. Math. Biol. 66, 1155-1171. doi: 10.1016/j.bulm.2003.11.005

Mittal, N., Zhou, Y., Ung, S., Linares, C., Molloi, S., and Kassab, G. S. (2005). A computer reconstruction of the entire coronary arterial tree based on detailed morphometric data. Ann. Biomed. Eng. 33, 1015-1026. doi: 10.1007/s10439-005-5758-z

Murray, C. D. (1926a). The physiological principle of minimum work: I. the vascular system and the cost of blood volume. Proc. Natl. Acad. Sci. U.S.A. 12, 207-214. doi: 10.1073/pnas.12.3.207

Murray, C. D. (1926b). The physiological principle of minimum work: II. oxygen exchange in capillaries. Proc. Natl. Acad. Sci. U.S.A. 12, 299-304. doi: $10.1073 /$ pnas.12.5.299

Namani, R., Kassab, G. S., and Lanir, Y. (2018). Integrative model of coronary flow in anatomically based vasculature under myogenic, shear, and metabolic regulation. J. Gen. Physiol. 150, 145-168. doi: 10.1085/jgp.201711795

Pries, A. R., and Secomb, T. W. (2009). Origins of heterogeneity in tissue perfusion and metabolism. Cardiovasc. Res. 81, 328-335. doi: 10.1093/cvr/cvn318

Pries, A. R., Secomb, T. W., and Gaehtgens, P. (1996). Biophysical aspects of blood flow in the microvasculature. Cardiovasc. Res. 32, 654-667. doi: 10.1016/S0008-6363(96)00065-X

Schindelin, J., Arganda-Carreras, I., Frise, E., Kaynig, V., Longair, M., Pietzsch, T., et al. (2012). Fiji: an open-source platform for biological-image analysis. Nat. Methods 9, 676-682. doi: 10.1038/nmeth.2019

Schindler, T. H. (2016). Myocardial blood flow: putting it into clinical perspective. J. Nucl. Cardiol. 23, 1056-1071. doi: 10.1007/s12350-015-0372-4
Schreiner, W., Karch, R., Neumann, M., Neumann, F., Roedler, S. M., and Heinze, G. (2003). Heterogeneous perfusion is a consequence of uniform shear stress in optimized arterial tree models. J. Theor. Biol. 220, 285-301. doi: $10.1006 /$ jtbi.2003.3136

Schreiner, W., Karch, R., Neumann, M., Neumann, F., Szawlowski, P., and Roedler, S. (2006). Optimized arterial trees supplying hollow organs. Med. Eng. Phys. 28, 416-429. doi: 10.1016/j.medengphy.2005.07.019

Smith, N. P., Pullan, A. J., and Hunter, P. J. (2000). Generation of an anatomically based geometric coronary model. Ann. Biomed. Eng. 28, 14-25. doi: 10.1114/1.250

Sriram, K., Intaglietta, M., and Tartakovsky, D. M. (2014). Hematocrit dispersion in asymmetrically bifurcating vascular networks. Am. J. Physiol. Heart Circ. Physiol. 307, H1576-H1586. doi: 10.1152/ajpheart.00283.2014

Stoll, M., Quentin, M., Molojavyi, A., Thamer, V., and Decking, U. K. (2008). Spatial heterogeneity of myocardial perfusion predicts local potassium channel expression and action potential duration. Cardiovasc. Res. 77, 489-496. doi: $10.1093 / \mathrm{cvr} / \mathrm{cvm} 060$

Strahler, A. N. (1957). Quantitative analysis of watershed geomorphology. Am. Geophys. Union Trans. 38, 913-920. doi: 10.1029/TR038i006p 00913

Tamaddon, H., Behnia, M., and Kritharides, L. (2016). A new approach to blood flow simulation in vascular networks. Comput. Methods Biomech. Biomed. Engin. 19, 673-685. doi: 10.1080/10255842.2015.1058926

Tange, O. (2011). GNU Parallel - The Command-Line Power Tool. Berkeley, CA: The USENIX Magazine.

VanBavel, E., and Spaan, J. A. (1992). Branching patterns in the porcine coronary arterial tree. Estim. Flow Heterog. Circ. Res. 71, 1200-1212. doi: 10.1161/01.RES.71.5.1200

Womersley, J. R. (1955). Oscillatory flow in arteries: effect of radial variation in viscosity on rate of flow. J. Physiol. 127, 38P-39P.

Womersley, J. R. (1958). Oscillatory flow in arteries. III. Flow and pulse-velocity formulae for a liquid whose viscosity varies with frequency. Phys. Med. Biol. 2, 374-382. doi: 10.1088/0031-9155/2/4/307

Yang, J., and Wang, Y. (2013). Design of vascular networks: a mathematical model approach. Int. J. Numer. Method. Biomed. Eng. 29, 515-529. doi: $10.1002 / \mathrm{cnm} .2534$

Yong, A. S. C., Javadzadegan, A., Fearon, W. F., Moshfegh, A., Lau, J. K., Nicholls, S., et al. (2017). The relationship between coronary artery distensibility and fractional flow reserve. PLOS ONE 12:e0181824. doi: 10.1371/journal.pone.0181824

Zamir, M. (1976a). Optimality principles in arterial branching. J. Theor. Biol. 62, 227-251. doi: 10.1016/0022-5193(76)90058-8

Zamir, M. (1976b). The role of shear forces in arterial branching. J. Gen. Physiol. 67, 213-222. doi: 10.1085/jgp.67.2.213

Zamir, M., and Phipps, S. (1988). Network analysis of an arterial tree. J. Biomech. 21, 25-34. doi: 10.1016/0021-9290(88)90188-1

Zamir, M., Wrigley, S. M., and Langille, B. L. (1983). Arterial bifurcations in the cardiovascular system of a rat. J. Gen. Physiol. 81, 325-335. doi: 10.1085/jgp.81.3.325

Zhang, J. M., Zhong, L., Luo, T., Huo, Y., Tan, S. Y., Wong, A. S., et al. (2014). Numerical simulation and clinical implications of stenosis in coronary blood flow. Biomed Res. Int. 2014:514729. doi: 10.1155/2014/514729

Conflict of Interest Statement: The authors declare that the research was conducted in the absence of any commercial or financial relationships that could be construed as a potential conflict of interest.

Copyright (C) 2018 Kharche, So, Salerno, Lee, Ellis, Goldman and McIntyre. This is an open-access article distributed under the terms of the Creative Commons Attribution License (CC BY). The use, distribution or reproduction in other forums is permitted, provided the original author(s) and the copyright owner are credited and that the original publication in this journal is cited, in accordance with accepted academic practice. No use, distribution or reproduction is permitted which does not comply with these terms. 